\title{
METASTATIC MALIGNANT MELANOMA OF SKIN
}

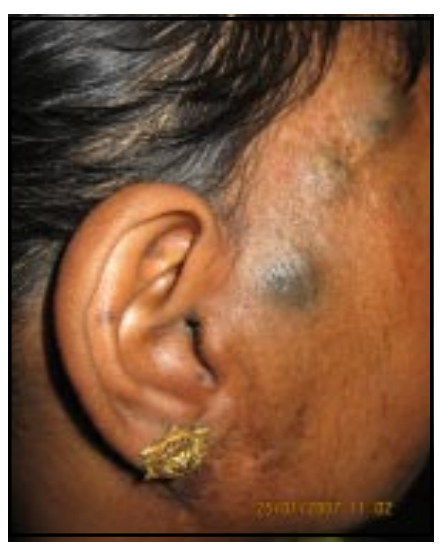

Fig-1 Blackish nodular lesions on pretemporal region suggestive of malignant melanoma

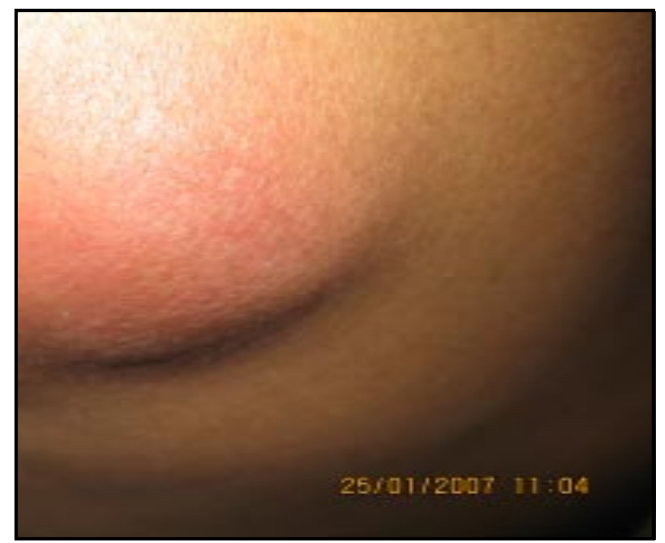

Fig-2. Subcutaneous nodule on the shoulder

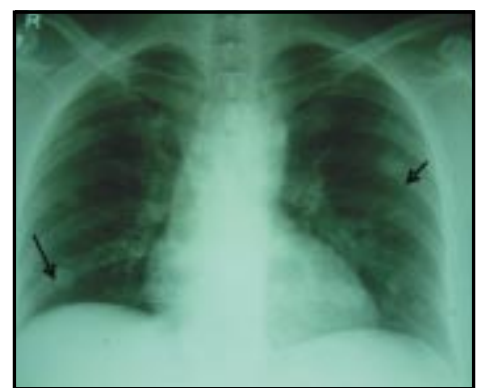

Fig: 3A Chest X-ray showing lung metastasis

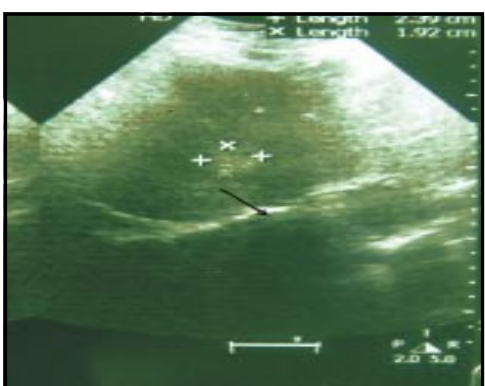

Fig: 3B USG abdomen showing liver metastasis

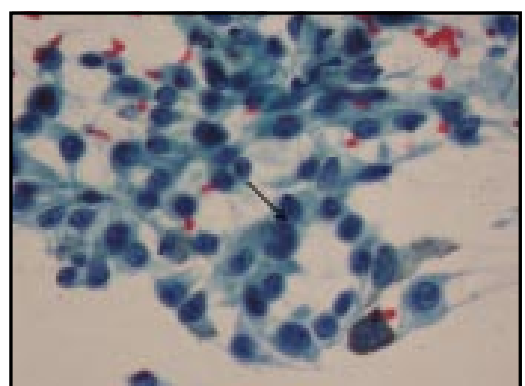

Fig:4 FNACof left scapular swelling showing tumour cells in discohesive group exhibiting prominent neucleolus and melanin pigment.-

Mrs. K.D, a 39 years old housewife had undergone local excision of a small blackish non tender nodule present on right side of forehead in 2004 and she presented to a private hospital in april 2006 with recurrence of same swellings and right sided facial palsy. Surgical excision of the preauricular nodule showed malignant melanoma and CAT scan revealed lobulated enhancing mass in the right parotid and temporal region. Chest X-ray and USG abdomen was suggestive of metastatic deposits in both lungs and liver. Post operatively, she received of combination chemotherapy with CVD (cisplatin, vinblastine, dacarbazine) and localized radiotherapy to these swellings. She presented with progressive disease in January 2007 (fig. 1, 2) Examination revealed residual facial palsy, skin lesions (fig:1), subcutaneous nodules in left shoulder (fig:2) and thigh and chest X-ray (fig 3A) and USG (fig:3B) of abdomen revealed metastasis. FNAC from skin nodule confirmed melanoma (Fig:4), she is on chemotherapy with CVD and continues to be stable.

Malignant melanoma is the most common skin cancer arising from the specialized pigmented cells known as melanocytes. Lung, liver, bone, brain are the common sites of metastasis. It is refractory to cytotoxic drugs and immunotherapies and has poor prognosis with a median survival of 6 months and 5 year survival of $5 \%$.

Aarti Gupta, Prashant G., Kamlesh Kumar* Sanjay Thulkar** Department of Medical Oncology, Pathology* \& Radiology** Institute Rotary Cancer Hospital All lIndia Institute of Medical Sciences New Delhi-110029 